\title{
Brittle Deformation of Solid and Granular Materials with Applications to Mechanics of Earthquakes and Faults
}

\author{
Yehuda Ben-Zion $^{1}$ and Charles Sammis ${ }^{1}$
}

\section{Introduction}

Crustal fault zones are complex regions of localized deformation with fractured, cataclastic and pulverized rocks having evolving geometries and altered rheological properties from those of the host material. At some level of fracture density (damage) the cohesive matrix of the material is destroyed and the associated volume becomes granular. The occurrence of seismic ruptures, and healing phases in the interseismic periods, continually modify the damage and granularity. This produces evolution of the elasticity, permeability and geometry of the actively deforming regions. The evolution of fault zone structures leads, in turn, to changes in the properties of dynamic earthquake ruptures, seismic radiation, inter- and post-seismic deformation, and local seismicity patterns.

Many fundamental aspects of brittle deformation in crustal rocks remain unsolved. Basic examples include: What are the proper metrics to characterize brittle rock damage and the transitions between damaged rock and granular material? What are the main similarities and differences between the dynamics of damaged rocks and granular media? What are the characteristics of nonlinear stress-strain behavior of damaged rocks and how can they be modeled quantitatively? How much slip is localized on main rupture surfaces and how much is distributed in the bulk for various fault environments? The 16 papers in this volume provide recent theoretical and observational perspectives that address the above and

1 Department of Earth Sciences, University of Southern California, Los Angeles, CA 90089-0740, USA. E-mail: benzion@usc.edu related issues. Topics include damage rheology models, high-resolution measurements of nonlinear evolving elasticity, high-resolution experiments on frictional instabilities and dynamic ruptures, theoretical and observational results on different dynamic regimes of sheared solids and granular materials, effects of fluids and roughness on fault zone rheology, seismic radiation near fault kinks, and geological and laboratory characterizations of fault surfaces and damaged rocks.

RUBINSTEIN et al. present laboratory results on slip along a frictional interface between two blocks loaded by shear at some height from the interface. Measurements of the evolving true contact area and loadings show sequences of progressively larger arrested rapid slip events and slow fronts. When these traverse the entire surface, dynamic system-size events occur. The observations are accompanied by theoretical analysis using block-spring model and discussion of results in the context of natural earthquakes. ARIAs et al. provide analytical results for singular static elastic field near a kink in a mode II shear crack. The solutions include power law functions with real exponents that depend on the angle of the kink, the coefficient of static friction and the difference of shear stress on the opposite sides of the corner. Differences of friction or loads across the corner can lead to tendencies to open the kink or close it more tightly.

ВнАт et al. present a generalized version of a micromechanical damage model based on tensile wing cracks that are nucleated and grow from a specified distribution of initial flaws. The model can explain observed nonlinearities in triaxial laboratory experiments with Westerly granite, and the dependence of strength on loading rate if the polymineralic nature of the granite is accounted for. HAmiel et al. discuss a 
second-order strain energy function of a continuum damage model that includes, in addition to the two regular terms of Hookean elasticity, a third non-analytic term. The latter accounts for nonlinear stress-strain relation, with abrupt changes of the effective elastic moduli upon stress reversal from compression to tension, along with damage- and stress-induced anisotropy. The model is compared with the third-order Murnaghan framework in the context of laboratory results associated with Westerly granite. TenCate reviews nonlinear resonance effects that characterize numerous geomaterials. Application of loadings above a certain threshold, which depends on the material and existing damage, leads to damage increase. In the absence of such loadings, the effective elastic properties recover with time following a $\log (t)$ functional form.

BEN-ZION et al. provide analytical mean-field results on different dynamic regimes of sheared solids and granular materials based on a model with long range interaction, evolving threshold dynamics and heterogeneities. The results are summarized in a phase diagram spanned by three tuning parameters. The basic dynamic regimes, seen in the response of both solid and granular materials to slow shear loading, are scale-invariant behavior, quasi-periodicity of systems size events, and long term mode-switching between these two types of response. Changes of cohesion lead to transitions between solid and granular states of material. HAYMAN et al. perform laboratory experiments with photoelasticity on stick-slip events in granular material. At relatively low packing of the material the frequency-size distribution of slip events is approximately power low, while at relatively high packing it is approximately exponential. Increasing packing fraction leads to more periodic behavior, but in all cases there is long-term switching between quasi-periodic and aperiodic responses. The aperiodic events involve major reorganizations of the force chains between particles. KRIM et al. analyze stick-slip events, steady sliding and inertial oscillations in laboratory experiments with a solid in contact with 2D photoelastic disks that are either fixed in a lattice (granular solid) or unconstrained (granular bed). The observed frictional properties depend on the form of contact, sliding speed, and applied vibrations. The effect of packing disorder in the case of granular bed appears similar to the effect of vibration induced disorder in the case of the granular solid.
Mair and Abe use a 3D discrete element model to simulate the microstructural evolution of fault gouge. Each grain is composed of several thousand spherical particles connected by breakable elastic bonds. Depending on the boundary conditions, their model produces two types of particle comminution: grain splitting which leads to a power law grain size distribution and abrasion which leads to a bimodal distribution. Splitting is favored at higher normal stress and rougher surfaces, while abrasion is favored by the opposite conditions. Goren et al. study the coupled mechanical behavior of granular material and fluid. For undrained conditions the behavior is elastic like, while for well-drained conditions it is viscous like. In addition to the known cases of liquefaction with high pore pressure and undrained conditions, liquefaction is shown to also occur for drained and initially over-compacted conditions. During liquefaction events, the stress chains are destroyed, the load is supported only by the pore fluid, and the shear resistance vanishes. SAmmis et al. show that the coefficient of friction of a layer of submicron particles can be strongly influenced by the properties of a few monolayers of absorbed water. At low slip speeds the particles have time to extrude the adsorbed layer and friction is controlled by rock-onrock contacts. At intermediate slip speeds there is not sufficient time to extrude the layer and friction drops. At high speeds sufficient heat is generated to vaporize the layer and friction returns to its rock-on-rock value. This explanation is shown to be in rough quantitative agreement with recent laboratory data.

ANGHEluta et al. use a simple model of a viscoelastic fault zone sandwiched between rigid wall rocks to explore the effect of surface roughness on the effective rheology of the layer. Analytical and numerical results indicate that the main effect of wall roughness is to increase the effective viscosity of the system. BistacCH et al. analyze roughness of slip surfaces exhumed from about $10 \mathrm{~km}$ in the strike-slip Gole Larghe fault zone in Italy. Using LIDAR and digital photography, the roughness is shown to be self-affine over 3-5 orders of magnitude. Results at scales smaller than the net fault slip are anisotropic implying evolution of roughness with slip. Differences of results for shallow and deeper faults are interpreted to reflect differences between weakening/ 
localization and induration/delocalization processes. Sмiтн et al. present detailed microstructural observations of slip zones in carbonates (limestone) associated with the Tre Monti normal fault in Italy. The principal slip zone has a $2-10 \mathrm{~mm}$ thick ultracataclasite immediately below the slip surface, with internal layering, calcite veins, fluidization structures and grains warped by calcite likely produced during seismic events, and overprinting foliation likely produced during interseismic periods. In the final two papers, HEESAKKERs et al. document the structure of the Pretorious fault at seismogenic depths of about $3 \mathrm{~km}$ where it intersects a deep mine in South Africa. Reactivation by a small mining-induced event caused slip on ancient and healed cataclasite localizations. Laboratory measurements of the strength of fault zone rocks and host rocks indicate that reactivation was due to the strong contrast in material properties between the cataclasites and host rock.

To conclude, earthquakes and fault mechanics involves brittle deformation of solids and granular materials, with a wide range of phenomena that operate over broad ranges of space and time scales. As reflected in the diverse papers collected here, insights into this rich field can come from many approaches ranging from statistical physics, structural geology and rock mechanics at large scales, elasticity and non-linear continuum mechanics at intermediate scales, and fracture mechanics, granular mechanics, and surface physics at small scales.

\section{Acknowledgments}

We thank the authors of the papers for their contributions and the referees for critical reviews that improved the scientific quality of the volume. The latter include Jean-Paul Ampuero, Gary Axen, Bob Behringer, Eran Bouchbinder, Brett Carpenter, Emily Brodsky, Fred Chester, Karin Dahmen, Karen Daniels, Steve Day, Eric Dunham, Yuri Fialko, Jay Fineberg, Yariv Hamiel, Nick Hayman, Karen Mair, Mike Mardar, Julia Morgan, Tom Mitchell, Stefan Nielsen, Hiroyuki Noda, Ze'ev Reches, Francois Renard, Amir Saggy, Norm Sleep, Heather Savage, Chris Scholz, Alexandre Schubnel, Leo Silbert, Steven Smith, Amy Rechenmacher, Thierry Reuschle and Renaud Toussaint. 